\title{
Correlates of fear of falling and falls efficacy in geriatric patients recovering from hip/pelvic fracture
}

Clinical Rehabilitation 2020, Vol. 34(3) 416-425

(C) The Author(s) 2019

Article reuse guidelines:

sagepub.com/journals-permissions

DOI: I0.1 I77/02692/55/989/233

journals.sagepub.com/home/cre

@SAGE

\author{
Tobias Eckert' ${ }^{(D)}$, Karin Kampe ${ }^{2}$, Michaela Kohler ${ }^{3}$, \\ Diana Albrecht ${ }^{3}$, Gisela Büchele ${ }^{4}, K^{\prime}$ laus Hauer', \\ Martina Schäufele ${ }^{5}$, Clemens Becker ${ }^{3}$ \\ and Klaus Pfeiffer ${ }^{3}$
}

\begin{abstract}
Objective: To gain a better understanding about the nature of fear of falling, this study analyzed associations between psychological and physical aspects related to fear of falling and falls efficacy in hip/ pelvic fracture patients.

Design: Baseline data of a randomized controlled trial.

Setting: Geriatric inpatient rehabilitation hospital.

Subjects: In all, II 5 geriatric patients with hip/pelvic fracture (mean age: 82.5 years) reporting fear of falling within first week of inpatient rehabilitation.

Interventions: None.

Main measures: Falls efficacy (Short Falls Efficacy Scale-International; Perceived Ability to Manage Falls), fear of falling (one-item question), fall-related post-traumatic stress symptoms (six items based on Diagnostic and Statistical Manual of Mental Disorders (4th ed.; DSM-IV) criteria), physical performance (Short Physical Performance Battery) and psychological inflexibility (Acceptance and Action Questionnaire-II) were assessed.

Results: Path analyses demonstrated that low falls efficacy (Short Falls Efficacy Scale International) was significantly related to poor physical performance $\left(\beta^{*}=-.277, P \leqslant .00 \mathrm{I}\right)$, but not to psychological inflexibility and fall-related post-traumatic stress symptoms $(P \geqslant .05$.). Fear of falling was directly associated with fallrelated post-traumatic stress symptoms $\left(\beta^{*}=.270, P=.007\right)$ and indirectly with psychological inflexibility $\left(\beta^{*}=.110, P=.022\right)$. Low perceived ability to manage falls was significantly related to previous falls $\left(\beta^{*}=-.348\right.$, $P \leqslant .00 \mathrm{I})$, psychological inflexibility $\left(\beta^{*}=-.216, P=.022\right)$ and female gender $\left(\beta^{*}=-.239, P \leqslant .0 \mathrm{I}\right)$.
\end{abstract}

\footnotetext{
'Agaplesion Bethanien Hospital Heidelberg, Geriatric Centre at the University of Heidelberg, Heidelberg, Germany 2Institute for Biomedicine of Aging, Friedrich-Alexander University Erlangen-Nürnberg, Erlangen, Germany ${ }^{3}$ Department of Clinical Gerontology and Geriatric Rehabilitation, Robert-Bosch-Hospital, Stuttgart, Germany ${ }^{4}$ Institute of Epidemiology and Medical Biometry, Ulm University, Ulm, Germany
}

\footnotetext{
${ }^{5}$ Department of Social Work, Mannheim University of Applied Sciences, Mannheim, Germany

Corresponding author:

Klaus Pfeiffer, Department of Clinical Gerontology and Geriatric Rehabilitation, Robert-Bosch-Hospital, Auerbachstr. I 10, Stuttgart D-70376, Germany.

Email: klaus.pfeiffer@rbk.de
} 
Conclusion: Falls efficacy and fear of falling constitute distinct constructs. Falls efficacy measured with the Short Falls Efficacy Scale International reflects the appraisal of poor physical performance. Fear of falling measured by the single-item question constitutes a fall-specific psychological construct associated with psychological inflexibility and fall-related post-traumatic stress symptoms.

\section{Keywords}

Hip fracture, elderly, fear of falling, falls efficacy, post-traumatic stress

Received: 21 December 2019; accepted: 7 November 2019

\section{Introduction}

Hip fractures are a major health issue for older adults. ${ }^{1}$ About $50 \%-68 \%$ of hip fracture inpatients report fear of falling ${ }^{2-4}$ and are at risk of poorer rehabilitation outcomes, ${ }^{5}$ the avoidance of physical activities and functional dependence. ${ }^{4}$

Because of different conceptualizations of fear of falling, there is some ambiguity in previous research. ${ }^{6}$ Based on Social Cognitive Theory, ${ }^{7}$ fear of falling originally has been conceptualized as "low perceived self-efficacy" in regard to beliefs and confidence about one's ability to avoid a fall. In more recent literature, the distinctiveness of fear of falling as an an and falls efficacy was underlined. ${ }^{6}$ In hip fracture patients, falls efficacy measured with the Falls Efficacy Scale International ${ }^{9}$ appears more closely associated with physical performance than to psychological constructs. ${ }^{10}$

The Perceived Ability to Manage Falls Scale ${ }^{11}$ is a further self-efficacy assessment that was found to be linked to generalized fear. ${ }^{11}$ Psychological aspects have strong associations to fear of falling measured with a single-item question. ${ }^{12}$ Fall-related post-traumatic stress symptoms are a possible affective consequence following a severe injurious fall. The reported prevalence as well as associations with fear of falling over the rehabilitation trajectory is inconsistent in previous research. ${ }^{13,14}$

Only little is known about emotion regulation processes underlying the different aspects of fear of falling. Psychological flexibility and inflexibility as a dimension of emotion regulation have received significant attention within clinical psychology during the last 20 years. Psychological inflexibility refers to the "rigid dominance of psychological reactions over chosen values and contingencies in guiding actions," 15 so that the person is not able to act in a flexible and context-sensitive manner. This construct has been found to be predictive for a wide range of psychological disorders and psychological distress caused by traumatic events. ${ }^{16}$

The aim of this study is a better understanding of the effects of physical performance and psychological inflexibility on different aspects of fear of falling with sex and previous falls as further predictors. Fall-related post-traumatic stress symptoms were considered as a possible mediator (Figure 1).

\section{Methods}

For the cross-sectional data analyses, we used baseline data of community-dwelling hip/pelvic fracture patients participating in a randomized, controlled intervention study to reduce fear of falling. ${ }^{17}$ The study protocol of this trial was approved by the ethics committee of the University of Tübingen and registered at www.isrctn.org (ISRCTN79191813). Data were collected between April 2011 and August 2013. Participants were consecutively recruited from a geriatric rehabilitation department of a German hospital. Main inclusion criteria were age $>60$ years, hip or pelvic fracture, the absence of cognitive impairment (Orientation-MemoryConcentration Test $\geqslant 10)^{18}$ and presence of fear of falling indicated by (a) frequent negative memories about the injurious fall, (b) concerns to fall in at least one specific activity during current recovery process after the fracture and (c) very concerned to fall again in the next year. Full data of recruitment and data collection procedure were published previously. ${ }^{17}$ For measuring fall-related concerns and fears, we used two different measures: A single-item question ("In general, are you afraid of falling?") 


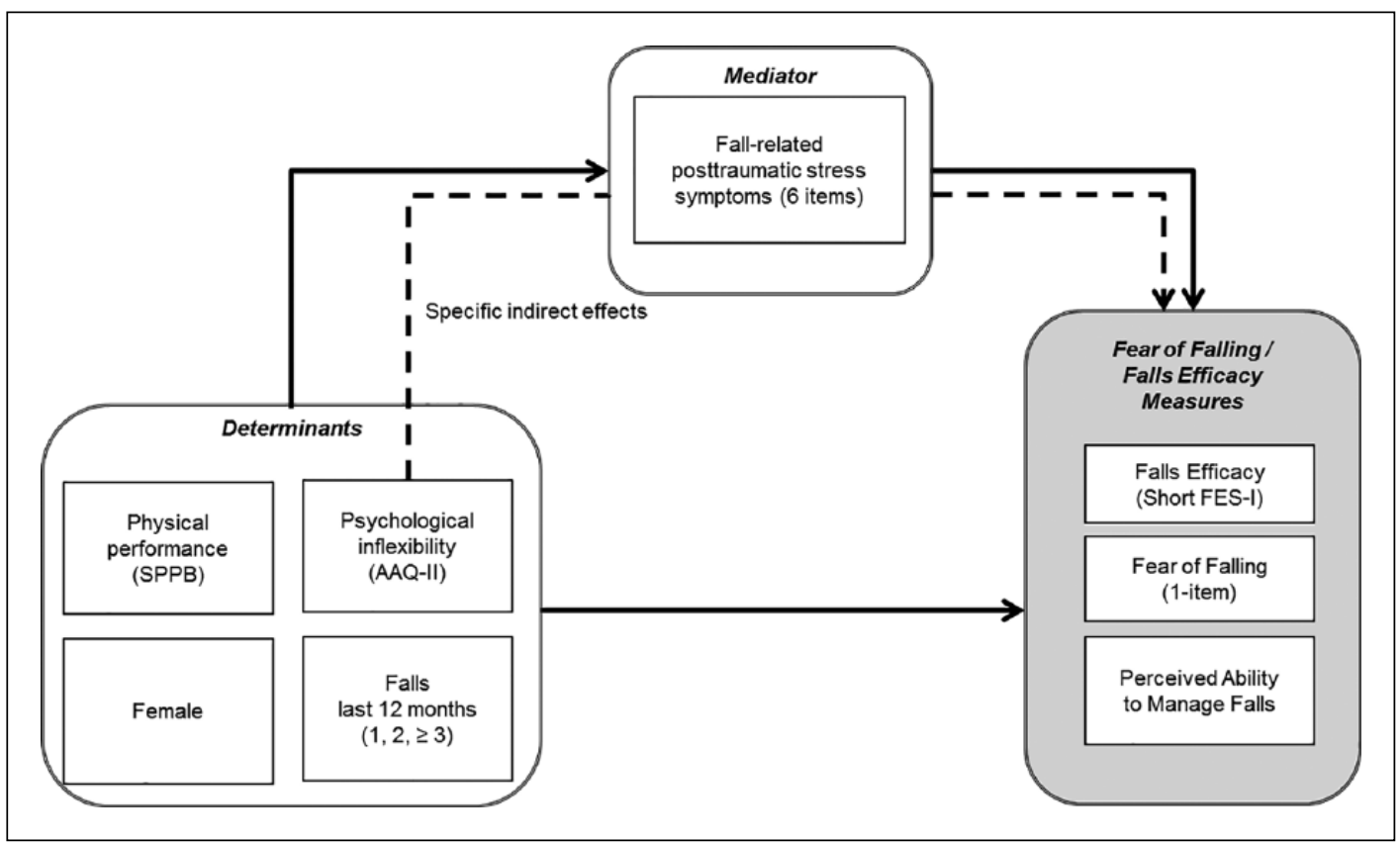

Figure I. Hypothesized path model to explore effects on fear of falling and falls efficacy.

Solid lines: direct effects; dotted lines: specific indirect effects; SPPB: Short Physical Performance Battery; AAQ-II: Acceptance and Action Questionnaire II; PAMF: Perceived Ability to Manage Falls; Short FES-I: Short Falls Efficacy Scale International.

with a 4-point Likert-type scale (scores from (0) "not at all," (1) "a little," (2) "quite a bit," (3) "very much") ${ }^{19}$ and the Short Fall-Efficacy Scale International (Short FES-I), which measures the level of concern about falling during social and physical activities inside and outside the home. ${ }^{9}$ Higher scores indicate higher levels of fall-related concerns. The internal consistency of the Short FES-I was good for our sample (Cronbach's $\alpha=.83$ ).

With the Perceived Ability to Manage Falls Scale, we measured self-efficacy components independent from specific physical activities. ${ }^{11}$ The five items capture the respondents' beliefs to avoid falls and to handle falls when they occur. Sum scores (4-20) with higher values reflect higher self-efficacy. Adequate internal consistency was shown within our sample (Cronbach's $\alpha=.66$ ).

Fall-related post-traumatic stress symptoms were assessed with six items based on criteria of the Diagnostic and Statistical Manual of Mental Disorders (4th ed.; DSM-IV), ${ }^{20}$ including four items from criteria B (re-experiencing the fall event): (a) recurrent and distressing recollections of the fall event, (b) distressing dreams of the fall event, (c) intense psychological distress and (d) physical reactivity at exposure to internal or external cues that symbolize an aspect of the fall event. The further two items from criteria $\mathrm{C}$ (avoidance of stimuli associated with the fall) were (e) efforts to avoid thoughts, feelings or conversations and (f) activities associated with the fall event. Responses were scored (0) "never," (1) "rather rarely," (2) "occasionally" and (3) "frequently." Sum score was used with a range from 0 to 18. Cronbach's alpha showed good internal consistency $(\alpha=.76)$.

Psychological inflexibility was assessed with the Acceptance and Action Questionnaire-II (AAQ-II). ${ }^{21,22}$ The seven items capture the respondents' unwillingness to deal with unpleasant emotions and experiences. Higher scores on AAQ-II are indicative for higher levels of psychological inflexibility with a total score range from 7 to 49 . Cronbach's alpha for the current sample proved excellent reliability $(\alpha=.89)$. 
Physical performance was assessed by the use of well-established and validated Short Physical Performance Battery (SPPB), including subtests for static balance, walking and sit-to-stand performance. Due to safety reasons, the patients were not asked to do the chairs stands "as quickly as possible." Habitual walking speed was assessed during one walk of a distance of $4 \mathrm{~m}$ as some of the hip and pelvic fracture patients could not perform two walks during early inpatient rehabilitation as prescribed in the original protocol. Performances of subtests were summarized to a sum score ranging from 0 to 12 with higher scores indicating a better physical performance. ${ }^{23}$

Participants' history of falls was measured by categorized number of falls within last 12 months via retrospective self-report including their fall causing the fracture ( 1 fall, 2 falls and $\geqslant 3$ falls). Falls were defined as "an unexpected event in which the participants come to rest on the ground, floor, or lower level." 24

With the Anxiety Subscale of the Hospital Anxiety and Depression Scale (HADS-A), ${ }^{25}$ an additional well-known measure was included in the descriptive and bivariate analyses to present associations with the AAQ-II and the other measures. The HADS-A includes seven items with a 4-point Likerttype scale and total scores from 0-21.

\section{Statistical analyses}

Associations between variables were calculated by Spearman's rank correlation coefficient $\left(r_{s}\right)$. The statistical analysis was conducted at a $95 \%$ confidence interval (CI); a $P$ value $<.05$ was considered statistically significant (two-sided). Basic analyses were conducted via IBM SPSS 24 Version.

The hypothesized path model was tested using Mplus Version 6.1. ${ }^{26}$ Due to the exploratory character of our model without a priori hypothesis on specific path associations, a saturated path analysis was derived. Therefore, fit indices are not reported. For estimating confidence limits and standard errors for indirect effect testing a non-bias-corrected bootstrap approach was used. ${ }^{27}$ An indirect effect was considered to be significant if its $95 \%$ bootstrap CIs from 10,000 bootstrap samples include zero or not. Standardized beta-weights $\left(\beta^{*}\right)$ and coefficients of determination $\left(R^{2}\right)$ are reported to describe direct and indirect effects and proportion of variance in the dependent variables that can be explained by the independent variables.

\section{Results}

\section{Participants}

Of the 759 patients with hip or pelvic fracture screened for eligibility, 115 were included in the study according to inclusion criteria (Figure 2). The present sample showed high functional restrictions and very low falls efficacy as indicated by high scores on the Short Falls Efficacy Scale International. According to previous cut-off points, ${ }^{28}$ two-thirds of our sample $(n=75 ; 65 \%)$ reported high concerns about falling (Short Falls Efficacy Scale International score $\geqslant 14$ ). In contrast, only $40 \%$ of the patients felt somewhat afraid or very afraid of falling when asked with the single-item question on fear of falling. Posttraumatic stress symptoms were also present within our cohort, since half of the participants $(n=66$; $49 \%)$ reported occasional $(n=33 ; 29 \%)$ or frequent $(n=23 ; 20 \%)$ recurrent and distressing recollections of the fall event. All descriptive measures are presented in Table 1.

\section{Correlations}

The results of bivariate correlation analyses among the variables are listed in Table 2. The measures on fear of falling and falls efficacy were significantly but solely moderately correlated. The highest correlation between these measures and physical performance was found for the Short Falls Efficacy Scale International. Psychological inflexibility was strongly linked to higher levels of fall-related post-traumatic symptoms and only very modestly with the three fear of falling measures. Both psychological inflexibility and fall-related post-traumatic stress symptoms were closely associated with anxiety. Non-fall-specific symptoms of anxiety were modestly correlated with the single-item question on fear of falling and the Short Falls Efficacy Scale International, but not with the Perceived Ability to Manage Falls scale. The latter 


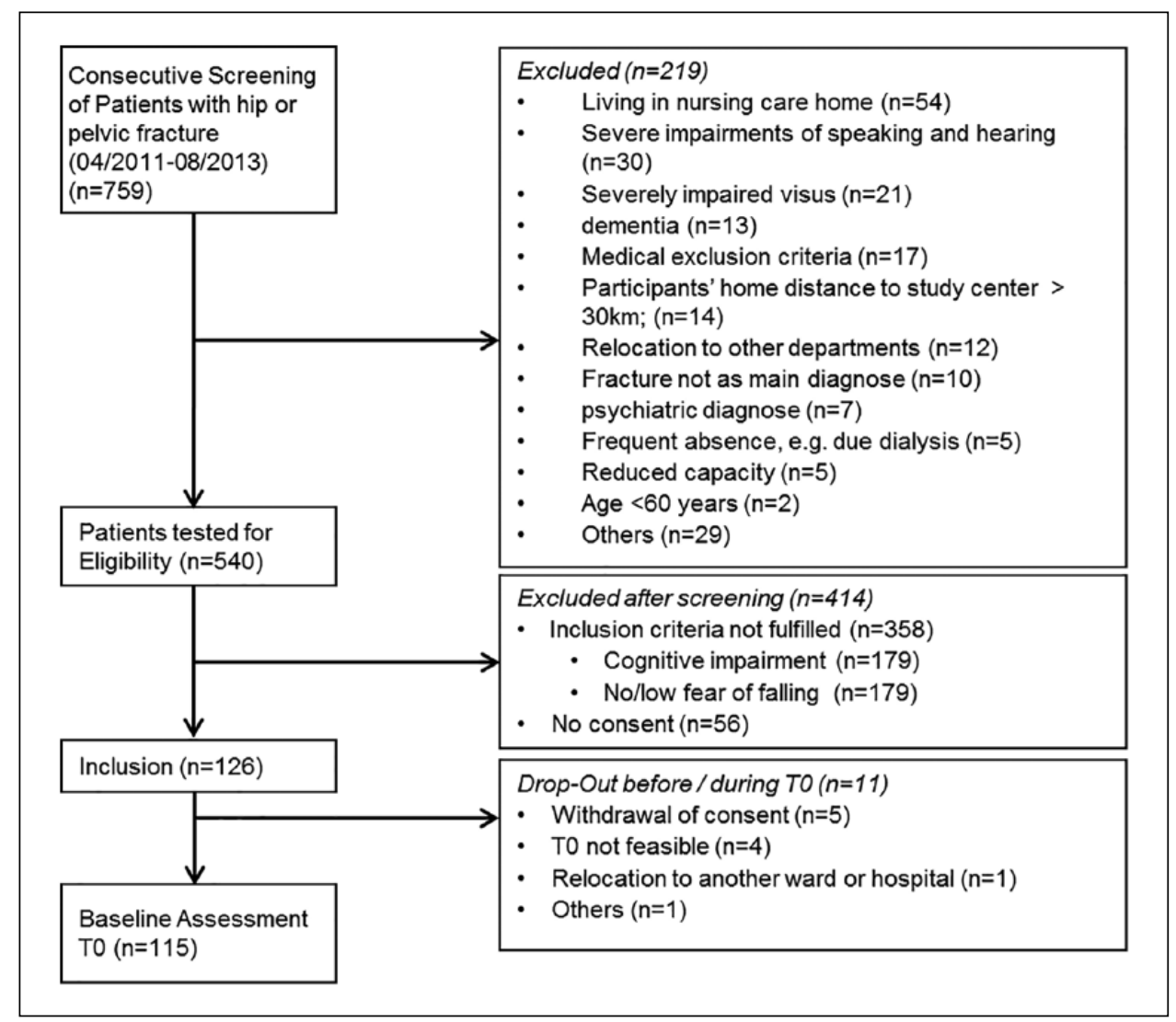

Figure 2. Participant flow in the study.

is the only measure that was significantly related to the number of previous falls.

\section{Path analysis}

The path analytic model (Figure 3) demonstrated that higher levels of fall-related concerns measured by the Short Falls Efficacy Scale International were only significantly determined by poor physical performance, but not by other psychological dimensions like increased fall-related post-traumatic stress symptoms and psychological inflexibility. In contrast, fear of falling showed a significant association to fall-related post-traumatic stress symptoms, which in turn was strongly affected by psychological inflexibility. In addition to these direct effects, we found a significant indirect effect of psychological inflexibility on fear of falling via post-traumatic stress symptoms.

Higher psychological inflexibility was associated with lower perceived ability to manage falls. In line with the bivariate analyses, females and individuals with multiple falls within the last year experienced significantly lower abilities to manage falls. Noteworthy, the proportion of variance explained of the dependent variables was lowest for the Short Falls Efficacy Scale International and highest for perceived ability to manage falls.

\section{Discussion}

This study demonstrated that low falls efficacy measured with the Short Falls Efficacy Scale International is primarily associated with poor 
Table I. Descriptive information about sociodemographic and clinical variables.

\begin{tabular}{lcc}
\hline Characteristic $(n=|| 5)$ & Mean (SD) & $N(\%)$ \\
\hline Age (years) & $82.5(6.8)$ & $87(76)$ \\
Gender: female & & $66(57)$ \\
Falls within last I2 months & & $23(20)$ \\
One fall (only the injurious fall) & & $26(23)$ \\
Two falls & & $21(18)$ \\
More than or equal to three falls & & $48(42)$ \\
One-item fear of falling & $34(30)$ \\
Not at all afraid & & $12(10)$ \\
Slightly afraid & & \\
Somewhat afraid & & \\
Very afraid & $15.8(4.9)$ & \\
Short FES-I & $5.1(4.2)$ & \\
PTSS & $12.9(2.3)$ & \\
PAMF & $19.0(7.3)$ & $3.0(2.0)$ \\
AAQ-II & $5.6(3.9)$ & \\
SPPB & & \\
HADS-A & & \\
\hline
\end{tabular}

AAQ-II: Acceptance and Action Questionnaire-II; HADS-A: Hospital Anxiety Depression Scale-Anxiety Subscale; PTSS = fallrelated post-traumatic stress symptoms; PAMF: perceived ability to manage falls; Short FES-I: Short Falls Efficacy Scale International; SPPB: Short Physical Performance Battery.

Table 2. Bivariate correlations between fear of falling (one-item question), falls efficacy (Short Falls Efficacy Scale International, Perceived Ability to Manage Falls), sociodemographic and clinical variables.

\begin{tabular}{llllllllll}
\hline Measure & 1 & 2 & 3 & 4 & 5 & 6 & 7 & 8 & 9 \\
\hline I. Age (years) & - & & & & & & & & \\
2. Gender (reference: male) & $.33^{* *}$ & - & & & & & & & \\
3. Falls (last I2 months) & .12 & -.08 & - & & & & & & \\
4. One-item FoF & .06 & .07 & .05 & - & & & & & \\
5. Short FES-I & .05 & .02 & .11 & $.45^{* *}$ & - & & & & \\
6. PTSS & .16 & .16 & .07 & $.39^{* *}$ & $.31^{* *}$ & - & & & \\
7. PAMF & -.13 & $-.22^{*}$ & $-.35^{* *}$ & $-.31^{* *}$ & $-.33^{* *}$ & $-.29^{* *}$ & - & & \\
8. AAQ-II & .05 & .06 & .02 & $.30^{* *}$ & $.28^{* *}$ & $.45^{* *}$ & $-.26^{* *}$ & - & \\
9. SPPB & .03 & .05 & -.14 & $-.30^{* *}$ & $-.37^{* *}$ & $-.20^{*}$ & $.23^{*}$ & $-.27^{* *}$ & - \\
I0. HADS-A & .04 & .11 & .03 & $.37^{* *}$ & $.31^{* *}$ & $.55^{* *}$ & -.17 & $.53^{* *}$ & $-.26^{* *}$ \\
\hline
\end{tabular}

AAQ-II: Acceptance and Action Questionnaire-II; HADS-A: Hospital Anxiety Depression Scale-Anxiety Subscale; PAMF:

Perceived Ability to Manage Falls; One-item FoF: one-item fear of falling; PTSS: fall-related post-traumatic stress symptoms; Short FES-I: Short Falls Efficacy Scale International; SPPB: Short Physical Performance Battery.

Level of significance $* p<.05 ; * * p<.01$.

physical performance in a sample of hip and pelvic fracture patients after admission to inpatient rehabilitation. In contrast, higher fear of falling is associated with other psychological dimensions like increased psychological inflexibility and fallrelated post-traumatic stress symptoms.

The percentage of persons with high fall-related concerns in our cohort $(65 \%)$ was comparable with 


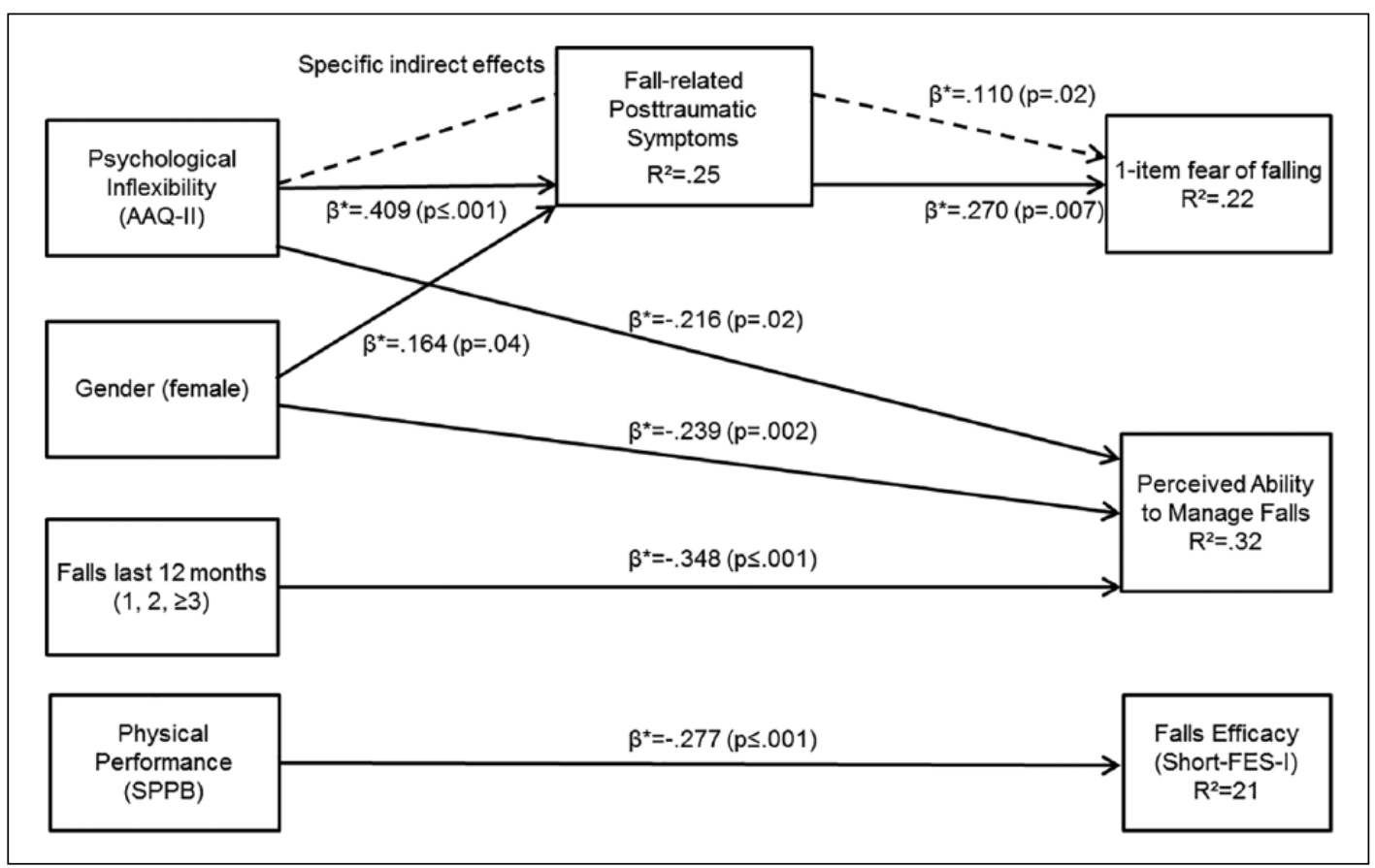

Figure 3. Results of the path analyses to examine fear of falling and falls efficacy including direct and indirect effects.

AAQ-II: Acceptance and Action Questionnaire-II; Short FES-I: Short Falls Efficacy Scale-International; SPPB: Short Physical

Performance Battery.

Significant total indirect effects from AAQ-II to Short FES-I $(P<.05)$ (not shown in the model) are presented.

Solid lines $=$ direct effects; dotted lines $=$ specific indirect effects.

$\beta^{*}$ : estimated values of standardized regression coefficients.

$R^{2}$ : squared multiple correlation coefficient indicating explained variance of endogeneous variables.

$* * * P<.001 ; * * P<.01 ; * P<.05$.

a previous study (62\%) using the 16-item Falls Efficacy Scale International at four weeks post surgery. ${ }^{2}$ In comparison to this previous study, fear of falling captured by the single item question was more often reported by the patients of the current study who were screened for at least some fear of falling symptoms ( $40 \%$ vs $27 \%$ ).

Noteworthy, there is a substantial difference between the levels of fear of falling measured by the Short Falls Efficacy Scale International and the single item question in the current study. In contrast to community-dwelling older adults, ${ }^{9}$ Short Falls Efficacy Scale International scores in our sample were also found to be high in individuals, who did report no or little fear of falling (data not shown here). This was especially true for those individuals with very low physical performance. For at least a subgroup of patients with high physiological risk of falling, concerns to fall may reflect the patients' awareness of their high actual fall risk. This realistic appraisal of one's high fall risk during this early period of rehabilitation can lead to low falls efficacy without having maladaptive fear of falling. This argumentation is in line with previous literature on perceived and physiological fall risk described for a sample of community-dwelling elderly. ${ }^{29}$

In contrast to the Short Falls Efficacy Scale International, fear of falling captured by a single item is rather linked to psychological correlates such as psychological inflexibility and fallrelated post-traumatic symptoms. This result confirms previous findings describing fear of 
falling measured with a one-item question as an anxiety reaction. ${ }^{28}$

Perceived ability to manage falls has been found to be associated with generalized fear in community-dwelling elderly. ${ }^{11}$ In our sample, the Perceived Ability to Manage Falls Scale had a lower internal consistency, but showed significant associations to previous falls, psychological inflexibility and sex. For those patients with low confidence to prevent and cope with falls, it seems important to provide knowledge on fall prevention and to promote fallrelated self-efficacy (e.g. fall prevention behavior, how to get up from the floor).

Previous research showed that older community dwellers using inflexible emotion regulation strategies like experiential avoidance more frequently reported higher levels of anxiety. ${ }^{30}$ Anxiety in turn was linked to fear of falling in community-dwelling older adults. ${ }^{31}$ Our study could show significant associations between psychological inflexibility and fall-related post-traumatic symptoms, fear of falling and perceived ability to manage falls. The avoidance of thoughts depicts also one core criterion of post-traumatic symptoms. Therefore, it is not surprising that psychological inflexibility seems to be predictive for post-traumatic symptoms, confirming results from studies in diverse populations after surviving a traumatic incident. ${ }^{16}$

With a few exceptions, emotion regulation has been widely disregarded in the fear of falling research. One recent study conducted in a non-clinical population found similar high bivariate associations between a more extensive emotion regulation assessment and anxiety measured with HADS like in our study ( $r=.47$ vs $r=.53$ in our study). The correlation between emotion regulation and the 16-item Falls Efficacy Scale International was slightly higher $(r=.36)$ in that community sample was compared to measures on the same domains in our inpatient sample $(r=.28){ }^{32}$ This comparison points in the same direction that emotion regulation in our sample is significantly less correlated with the Falls Efficacy Scale International rather than with anxiety. Experiential avoidance as an aspect of psychological inflexibility might be a factor in the downward spiral of activity avoidance and subsequent functional decline reported for hip fracture patients after hospital discharge. ${ }^{4}$ Improving the patient's ability to experience fall-related thoughts and fears without needless defense and avoidance could be a future addition of interest to recent cognitive behavioral approaches for older adults with fear of falling.

We also take account for the limitations of our study. This study used a selective group of hip fracture patients without significant cognitive impairment and at least some concerns about falling, so that findings are not generalizable for all hip and pelvic fracture patients. Despite the widespread use of the AAQ-II in both clinical and scientific contexts, a lack of discriminant validity is criticized. It is questioned whether the associations found between the AAQ-II as a measure of psychological inflexibility and measures related to psychological well-being are only due to a similar operationalization. ${ }^{16}$ Due to the lack of validated measures of fall-related post-traumatic stress symptoms, we used a self-developed symptom questionnaire based on DSM-IV criteria. In addition, the crosssectional design does not allow conclusions about robust causal associations and differences over the process of functional recovery.

The present results confirm the argumentation in previous conceptualizations ${ }^{6}$ that falls efficacy and fear of falling are different constructs. The close association between the Short Falls Efficacy Scale International and physical performance scores are in line with previous findings which demonstrated the suboptimal construct validity of the original 16-item version of the questionnaire in hip fracture patients. ${ }^{10}$ Furthermore, cut-off values of the Short Falls Efficacy Scale International found in community-dwelling elderly ${ }^{33}$ may not be adequate in more functionally restricted target groups. Fall-related post-traumatic symptoms were prevalent in at least a subgroup of hip and pelvic fracture patients and highly correlated with anxiety. The role of psychological constructs including anxiety, fall-related post-traumatic symptoms and psychological inflexibility for developing either a more generalized or a highly contextual fear of falling need further investigation. Furthermore, future empirical research testing the hypothesis that maladaptive fear of falling is always concurrent with anxiety while adaptive fear of falling is not $^{34}$ would be of interest in our target group. 
With this study, we have advanced the discussion for a better understanding of fall-related selfefficacy and fear of falling in hip and pelvic fracture patients after admission to rehabilitation. Psychological inflexibility seems to be a further facet in the puzzle and might have an effect on how hip and pelvic fracture patients cope with the fall event and its consequences. Assessments on fear of falling or falls efficacy in hip and pelvic fracture patients should always be interpreted with regard to the actual fall risk and activity avoidance due to fear of falling. Patients should be educated and supported in a realistic and context-sensitive fall risk appraisal and situation-specific behavior including a secure use of prescribed walking aids. Situationspecific behavior can include both asking or ringing the call bell and waiting for assistance in fall risk situations as well as coping with own maladaptive fears to benefit most from rehabilitation. ${ }^{35}$

\section{Clinical messages}

- Short Falls Efficacy Scale International scores are mainly associated with physical performance.

- Fall-related post-traumatic symptoms are common in hip and pelvic fracture patients and associated with fear of falling measured by the single item.

- Psychological inflexibility is highly correlated with anxiety and seems to be a predictor for fall-related post-traumatic symptoms, fear of falling and perceived ability to manage falls.

\section{Declaration of conflicting interests}

The author(s) declared the following potential conflicts of interest with respect to the research, authorship and/or publication of this article: C.B. has received consultation fees by E. Lilly and Bosch Health Care.

\section{Funding}

The author(s) disclosed receipt of the following financial support for the research, authorship and/or publication of this article: The study was funded by the German Federal Ministry of Education and Research (PROFinD, grant number 01EC1007A).

\section{ORCID iD}

Tobias Eckert (iD https://orcid.org/0000-0002-0565-7998

\section{References}

1. Requena G, Abbing-Karahagopian V, Huerta C, et al. Incidence rates and trends of hip/femur fractures in five European countries: comparison using e-healthcare records databases. Calcif Tissue Int 2014; 94(6): 580-589.

2. Visschedijk J, van Balen R, Hertogh C, et al. Fear of falling in patients with hip fractures: prevalence and related psychological factors. J Am Med Dir Assoc 2013; 14(3): 218-220.

3. Bower ES, Wetherell JL, Petkus AJ, et al. Fear of falling after hip fracture: prevalence, course, and relationship with one-year functional recovery. Am J Geriatr Psychiatry 2016; 24(12): 1228-1236.

4. Jellesmark A, Herling SF, Egerod I, et al. Fear of falling and changed functional ability following hip fracture among community-dwelling elderly people: an explanatory sequential mixed method study. Disabil Rehabil 2012; 34(25): 2124-2131.

5. Oude Voshaar RC, Banerjee S, Horan M, et al. Fear of falling more important than pain and depression for functional recovery after surgery for hip fracture in older people. Psychol Med 2006; 36(11): 1635-1645.

6. Hadjistavropoulos T, Delbaere K and Fitzgerald TD. Reconceptualizing the role of fear of falling and balance confidence in fall risk. J Aging Health 2011; 23(1): 3-23.

7. Bandura A. Self-efficacy: toward a unifying theory of behavioral change. Psychol Rev 1977; 84(2): 191-215.

8. Tinetti ME, Richman D and Powell L. Falls efficacy as a measure of fear of falling. J Gerontol 1990; 45(6): P239-P243.

9. Kempen GI, Yardley L, van Haastregt JC, et al. The Short FES-I: a shortened version of the falls efficacy scale-international to assess fear of falling. Age Ageing 2008; 37(1): 45-50.

10. Visschedijk J, Terwee CB, Caljouw MA, et al. Reliability and validity of the falls efficacy scale-international after hip fracture in patients aged $\geqslant 65$ years. Disabil Rehabil 2015; 37(23): 2225-2232.

11. Lawrence RH, Tennstedt SL, Kasten LE, et al. Intensity and correlates of fear of falling and hurting oneself in the next year. J Aging Health 1998; 10(3): 267-286.

12. Jorstad EC, Hauer K, Becker C, et al. Measuring the psychological outcomes of falling: a systematic review. $J \mathrm{Am}$ Geriatr Soc 2005; 53(3): 501-510.

13. Chung MC, McKee KJ, Austin C, et al. Posttraumatic stress disorder in older people after a fall. Int $J$ Geriatr Psychiatry 2009; 24(9): 955-964.

14. Kornfield SL, Lenze EJ and Rawson KS. Predictors of posttraumatic stress symptoms and association with fear of falling after hip fracture. J Am Geriatr Soc 2017; 65: 1251-1257. 
15. Hayes SC, Luoma JB, Bond FW, et al. Acceptance and commitment therapy: model, processes and outcomes. Behav Res Ther 2006; 44(1): 1-25.

16. Wolgast M. What does the Acceptance and Action Questionnaire (AAQ-II) really measure? Behav Ther 2014; 45(6): 831-839.

17. Kampe K, Kohler M, Albrecht D, et al. Hip and pelvic fracture patients with fear of falling: development and description of the "Step by Step" treatment protocol. Clin Rehabil 2017; 31(5): 571-581.

18. Katzman R, Brown T, Fuld P, et al. Validation of a short orientation-memory-concentration test of cognitive impairment. Am J Psychiatry 1983; 140: 734-739.

19. Yardley L and Smith H. A prospective study of the relationship between feared consequences of falling and avoidance of activity in community-living older people. Gerontologist 2002; 42(1): 17-23.

20. $\mathrm{Sa} ß \mathrm{H}$ (ed.). Diagnostisches und statistisches Manual psychischer Störungen: textrevision; DSM-IV-TR (übersetzt nach der Textrevision der 4. Aufl, XXIV, $1001 \mathrm{~S}$ ). Göttingen; Bern: Hogrefe, 2003.

21. Bond FW, Hayes SC, Baer RA, et al. Preliminary psychometric properties of the Acceptance and Action QuestionnaireII: a revised measure of psychological inflexibility and experiential avoidance. Behav Ther 2011; 42(4): 676-688.

22. Hoyer J and Gloster AT. Psychologische Flexibilität messen: Der Fragebogen zu Akzeptanz und Handeln II. Verhaltenstherapie 2013; 23(1): 42-44.

23. Guralnik JM, Simonsick EM, Ferrucci L, et al. A short physical performance battery assessing lower extremity function: association with self-reported disability and prediction of mortality and nursing home admission. $J$ Gerontol 1994; 49(2): M85-M94.

24. Lamb SE, Jorstad-Stein EC, Hauer K, et al. Development of a common outcome data set for fall injury prevention trials: the Prevention of Falls Network Europe consensus. J Am Geriatr Soc 2005; 53(9): 1618-1622.
25. Zigmond AS and Snaith RP. The hospital anxiety and depression scale. Acta Psychiatr Scand 1983; 67(6): 361-370.

26. Muthén L and Muthén B. Mplus software (version 6). Los Angeles, CA: Muthén \& Muthén, 2010.

27. Fritz MS, Taylor AB and Mackinnon DP. Explanation of two anomalous results in statistical mediation analysis. Multivariate Behav Res 2012; 47(1): 61-87.

28. Yardley L. Fear of imbalance and falling. Rev Clin Gerontol 1998; 8(1): 23-29.

29. Delbaere K, Close JC, Brodaty H, et al. Determinants of disparities between perceived and physiological risk of falling among elderly people: cohort study. BMJ 2010; 341: c4165.

30. Andrew D and Dulin PL. The relationship between selfreported health and mental health problems among older adults in New Zealand: experiential avoidance as a moderator. Aging Ment Health 2007; 11(5): 596-603.

31. Hull SL, Kneebone II and Farquharson L. Anxiety, depression, and fall-related psychological concerns in community-dwelling older people. Am J Geriatr Psychiatry 2013; 21(12): 1287-1291.

32. Scarlett L, Baikie E and Chan SWY. Fear of falling and emotional regulation in older adults. Aging Ment Health 2019; 23: 1684-1690.

33. Delbaere K, Close JC, Mikolaizak AS, et al. The falls efficacy scale international (FES-I). A comprehensive longitudinal validation study. Age Ageing 2010; 39(2): 210-216.

34. Adamczewska N and Nyman SR. A new approach to fear of falls from connections with the posttraumatic stress disorder literature. Gerontol Geriatr Med 2018; 4: 2333721418796238.

35. Hill AM, McPhail SM, Waldron N, et al. Fall rates in hospital rehabilitation units after individualised patient and staff education programmes: a pragmatic, steppedwedge, cluster-randomised controlled trial. Lancet 2015; 385(9987): 2592-2599. 